\title{
Effect of Vaneless Diffuser Width on the Overall Performance of a
}

\section{Centrifugal Compressor}

\author{
Ahti Jaatinen, Aki Grönman, Teemu Turunen-Saaresti and Pekka Röyttä \\ Institute of Energy, Lappeenranta University of Technology, P.O. Box 20, FI-53851 Lappeenranta, \\ Finland
}

Abstract: Seven different vaneless diffuser designs varying in diffuser width are studied experimentally. One design is the basic design with the diffuser width and impeller exit and tip clearance width ratio of 1.0. The other six diffusers have width ratios of $0.903,0.854$, and 0.806 . Three of the narrower diffusers have the width reduced from the shroud, and three from the hub and the shroud, divided evenly. The total-to-total efficiency and pressure ratio over the whole compressor are studied. It is possible to increase the efficiency of the compressor stage by reducing the diffuser width. The efficiency is increased over a wide operating range area at three different rotational speeds. The pressure ratios are increased at the design and low rotational speeds, but decreased at the high rotational speed. The shroud pinch seems to be more beneficial to the performance, while the hub pinch seems to have only a minor effect. The best design is the one with the width ratio of 0.854 , with width reduction only at the 
shroud. A major finding is the experimental confirmation that the pinch influences the performance of the impeller, reducing the work input at the higher rotational speed.

Keywords: centrifugal compressor, vaneless diffuser, diffuser width, efficiency

\section{INTRODUCTION}

The motivation for improving the performance of a centrifugal compressor is a decrease in electricity consumption, which will lead to lower emissions in power generation. Certain industrial applications where centrifugal compressors are used, e.g. municipal waste water treatment, prefer a wider operating range in order to avoid installing additional compressor units. Vaneless diffusers are used in applications where an advantageous price and a wide operating range are the main goals.

However, today's need for more energy- efficient processes demands that efficiency is one of the main targets also in these applications.

A vaneless diffuser is a simple geometry consisting of two parallel or almost parallel walls. Pinch means that the diffuser width is decreased from the width of the impeller exit and the tip clearance. The reduction of width can be made to the hub, the shroud, or both walls.

The most important design criteria affecting the performance of vaneless diffusers are the channel width and the radius ratio of the impeller outlet and the diffuser outlet. Diffuser width has a very significant effect on the critical flow angle [1]. The critical flow angle is the most tangential angle with which the diffuser does not stall. The smaller the diffuser width, the more tangential is the critical flow angle. Similar results are also presented in [2], and this is the case for centrifugal blowers as well [3].

Four different vaneless diffusers were studied experimentally in [4]. The diffusers were parallel wall, highly tapered, constant area, and parallel wall with the width ratio $b / b_{2}=0.53$. The author 
found that the parallel wall diffuser with reduced width had a lower choke limit and a lower surge limit. The efficiency was reduced when compared to the parallel wall diffuser with no pinch, but the size of pinch was rather high. He also noted that reducing the diffuser width shifted the maximum efficiency points towards respective surge points.

Diffusers with two different diffusion ratios, three different diffuser widths $\left(b / b_{2}=0.30,0.38\right.$ and 0.64), and three different pinch shapes: one with a quarter of circumference as the inlet profile, one with an almost linear inlet shape, and one with an elliptical arc as the inlet profile were tested in $[5,6,7]$

The diffusers were tested for two different high pressure impellers. It was found that the pinch shape had very little or no effect on the stall inception or the stage performance.

For the higher diffusion ratio, reducing the diffuser width decreased the critical flow angle, and vice versa for the lower diffusion ratio. Decreasing the diffuser width led to lower efficiencies when compared to the diffuser with the width ratio $b / b_{2}=0.64$. The efficiency reductions were from $2 \%$ to $13 \%$. The reduction in the diffuser width led to higher pressure losses in the diffuser, even though the maximum head coefficients increased by $4-9 \%$. The results were similar for both impellers.

Two parallel wall vaneless diffusers with different widths were tested in [8]. The diffuser widths were 9.40 and $11.18 \mathrm{~mm}$. Unfortunately, the impeller exit width was not reported. The authors found that the narrower of the two diffusers had slightly better efficiency. It also had a somewhat better total-to-total pressure ratio, except at the low speed, where almost identical performance was obtained for both diffusers. It was also noted that the diffuser width did not influence the impeller performance.

In [9], unsteady measurements for two different diffuser widths were done, and it was found that with the increasing diffuser width, the onset of the impeller rotating stall shifted to lower flow rates, and the frequencies of the rotating stalls decreased. 
Previous numerical results [10] suggest that a pinched diffuser has better efficiency than an unpinched one. Also the pressure ratio is slightly increased. These numerical results also show that the best performance is obtained when the hub and the shroud walls are pinched. The impeller tip clearance was not modelled in this study.

Pinch increases the radial velocity in the diffuser. If the increase in the radial velocity is the defining parameter, the effect of pinch should be greater at a higher rotational speed, and lower at a lower rotational speed. The effect should also be grater at lower mass flows than at the design mass flow.

In this paper, seven different vaneless diffuser geometries with diffuser width ratios varying from $b / b_{2}=1$ to $b / b_{2}=0.806$ are investigated experimentally. Three of the geometries have pinch both at the hub and at the shroud, divided evenly, three have pinch only at the shroud, and one is unpinched.

The literature survey did not support the idea that the efficiency can be improved with pinch. However, the reductions in diffuser widths reported in the literature seem to be quite large. We study smaller pinches and try to find out if there is an optimum reduction.

The first major result of this paper is that it is possible to improve compressor stage efficiency and pressure ratio by reducing the diffuser width, if the reduction is moderate. The second major result is the experimental confirmation that the pinch influences the impeller performance. This is seen as a reduction of work input in the impeller at a higher rotational speed. It is very plausible that this occurs also at the design and lower rotational speeds. The reduction in the diffuser width is relatively easy to design and implement to manufacturing, giving quite an easy way to conserve energy in process industry. 


\section{MEASUREMENTS}

\subsection{Measured designs}

Six pinched geometries and one unpinched geometry were measured. Three diffusers with shroud pinch, and three with hub and shroud pinches were measured. For the pinched diffusers, the diffuser width ratio was varied from $b / b_{2}=0.903$ to $b / b_{2}=0.806$.

The diffuser height was constant over the diffuser length. The pinch began at the constant radius ratio $r_{2}, / r_{2}=1.01$ in all pinched geometries. The ratio of the diffuser exit and impeller exit radii was a constant 1.68 in all geometries. A summary of the studied configurations are shown in Table 1 . The second diffuser in the table $\left(b / b_{2}=0.903\right.$ hub $\&$ shroud $)$ has the same amount of pinch in the shroud that what is the impeller tip clearance at the impeller outlet.

Table 1. The studied designs.

\begin{tabular}{cccl}
\hline Case & $b / b_{2}$ & $b / r_{2}$ & Pinch \\
\hline 1 & 1.000 & 0.1217 & Unpinched \\
2 & 0.903 & 0.1098 & Hub \& shroud \\
3 & 0.854 & 0.1039 & Hub \& shroud \\
4 & 0.806 & 0.0979 & Hub \& shroud \\
5 & 0.903 & 0.1098 & Shroud \\
6 & 0.854 & 0.1039 & Shroud \\
7 & 0.806 & 0.0979 & Shroud \\
\hline
\end{tabular}

The positions of the different stations are the following: station 1 is the compressor inlet, 2 is the impeller outlet, $2^{\prime}$ is the beginning of pinch, 3 is the diffuser outlet, and station 5 is the compressor outlet. The schematics of the pinch are presented in Figure 1. 


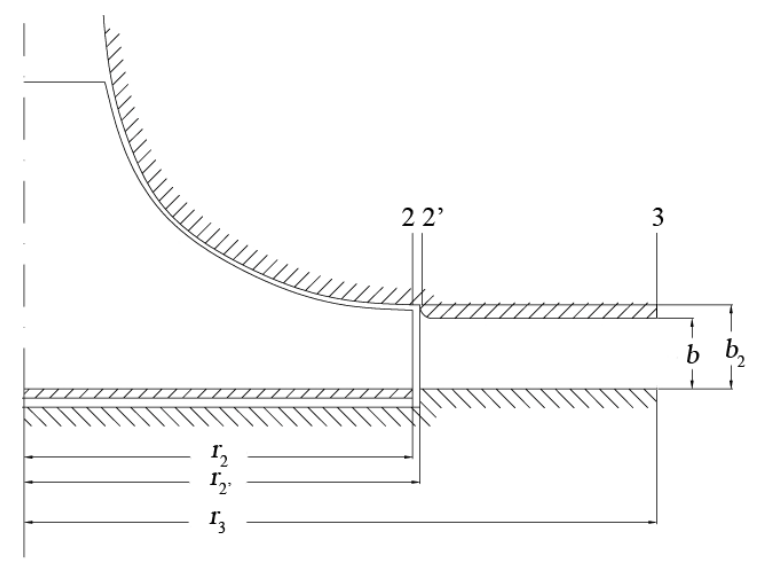

Figure 1. Schematics of the pinch.

\subsection{Measurement setup}

The experiments were conducted at a compressor test stand in the Laboratory of Fluid Dynamics at Lappeenranta University of Technology. The test facility consists of a one-stage industrial centrifugal compressor. The compressor is driven directly by a high-speed electric motor, and the rotational speed is adjusted with a variable-speed drive.

\subsubsection{Test compressor}

The compressor design pressure ratio $\pi_{\mathrm{tt}}$ is 1.78 , and the specific rotational speed, defined as

$$
N_{s}=\frac{\Omega \sqrt{q_{v}}}{\Delta h_{s}^{0.75}}
$$

is 0.8 . The test compressor impeller has seven full and seven splitter blades with a $40^{\circ}$ back sweep from the radial direction.

The ratio of the rotor outlet height to the outlet radius $b_{2} / r_{2}$ is 0.1214 , and the ratio of the diffuser outlet radius to the rotor outlet radius $r_{3} / r_{2}$ is 1.681 for the original vaneless diffuser. The diffuser height is constant over the diffuser length. 
The test compressor is equipped with a volute. The volute is designed to provide constant circumferential pressure distribution at the design mass flow. An exit cone is placed after the volute in order to mount the compressor stage to standard piping.

\subsubsection{Test stand}

The efficiency and pressure ratio of the compressor stage are measured by using the pressure and temperature measurements of the test stand. The air enters the compressor through a mass flow nozzle, flow straightener and throttling valve. The mass flow nozzle is an ISA 1932 nozzle made according to the DIN 1952 standard [11]. The performance calculations of the compressor and the instrumentation of the test stand are carried out according to standards $[12,13,14,15]$. The layout of the test stand is shown in Fig. 1.

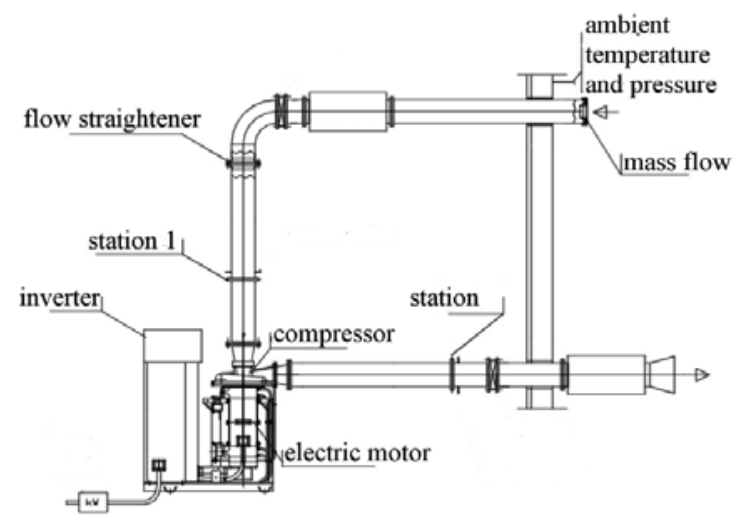

Figure 2. Layout of the test stand.

\subsection{Measurement procedure}

In the overall performance measurements of the compressor, the compressor was insulated to achieve better accuracy. For each geometry, the measurements began at the lowest rotational speed, and after a given rotational speed curve was measured, the rotational speed was increased.

For a given rotational speed, the measurements began at the choke. The compressor was left to run until the temperatures had settled, and then a point was recorded. This was repeated until low 
mass flows were reached. In total of 5-13 different mass flow points were measured for each rotational speed.

For the high rotational speed, at high mass flows near the choke, the limiting factor was the cooling of the electric motor running the compressor, so these points were not measured, or they were measured quicker.

When the results were processed, eight consecutive measurements were taken for a given operating point and the average value was given as a single operating point. The maximum relative measurement uncertainties for the mass flow, efficiency and pressure ratio in the overall performance measurements with the $95.4 \%$ confidence interval were:

$$
\begin{aligned}
& E_{\mathrm{qm}, 95.4}= \pm 0.17 \% \\
& E_{\eta, 95.4}= \pm 0.47 \% \\
& E_{\pi, 95.4}= \pm 0.33 \%
\end{aligned}
$$

\section{MEASUREMENT RESULTS}

The measurement station intakes atmospheric air. The atmospheric conditions may vary during and between the measurements, so the measured values must be converted to reference values. This ensures that the performance measurements done under different conditions are comparable with each other.

The conversion to reference values is done with applying the reference total pressure $p_{\mathrm{t} 1 \text {, ref }}=$ $101.3 \mathrm{kPa}$, the reference total temperature $T_{\mathrm{t} 1, \text { ref }}=288 \mathrm{~K}$, and the reference relative humidity $R_{\mathrm{H}}=$ $60 \%$. The reference relative humidity is used to calculate the specific gas constant $R$.

The above-mentioned reference values are then used to calculate the reference mass flow $q_{\mathrm{m}, \mathrm{ref}}$ and the reference rotational speed $N_{\text {ref, }}$ which are calculated as

$$
q_{\mathrm{m}, \mathrm{ref}}=q_{\mathrm{m}} \frac{p_{\mathrm{t} 1, \mathrm{ref}}}{p_{\mathrm{t} 1}} \sqrt{\frac{T_{\mathrm{t} 1} R}{T_{\mathrm{t} 1, \mathrm{ref}} R_{\mathrm{ref}}}}
$$




$$
N_{\text {ref }}=N \sqrt{\frac{T_{\mathrm{t} 1, \mathrm{ref}} R_{\mathrm{ref}}}{T_{\mathrm{t} 1} R}}
$$

\subsection{Efficiency}

The total-to-total isentropic efficiency over the compressor stage is defined as

$$
\eta_{\mathrm{tt}, 15}=\frac{T_{\mathrm{ts} 5}-T_{\mathrm{t} 1}}{T_{\mathrm{t} 5}-T_{\mathrm{t} 1}}
$$

where the total isentropic temperature is a function of the pressure ratio

$$
T_{\mathrm{ts} 5}=T_{\mathrm{t} 1} \pi_{\mathrm{tt}, 15}^{\frac{R}{c_{\mathrm{p}}}}
$$

The total-to-total isentropic efficiencies at the design rotational speed, nondimensionalized with the design efficiency, are presented in Fig. 2 for the hub and shroud pinches, and in Fig. 3 for the shroud pinches.

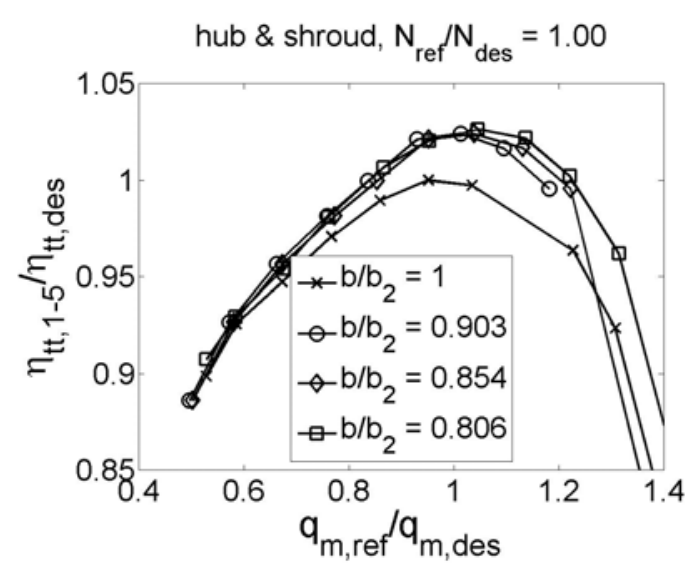

Figure 3. Total-to-total stage efficiency at the design rotational speed (hub and shroud pinches). 


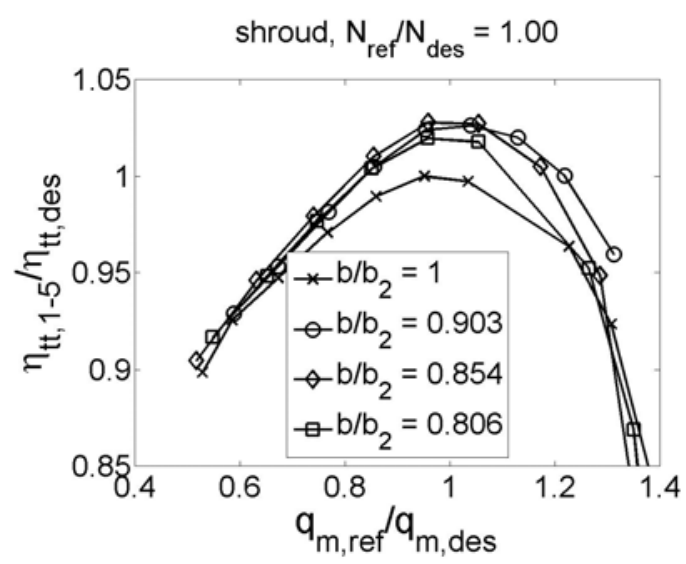

Figure 4. Total-to-total stage efficiency at the design rotational speed (shroud pinches).

The pinched geometries have clearly higher efficiency than the unpinched one. The best design with the hub and shroud pinch is the one with the diffuser width ratio of 0.806 , and with the shroud pinch the one with the diffuser width ratio of 0.854 . The differences between the different pinched geometries are minor when compared with their difference to the unpinched design.

With the pinched designs, the peak efficiency is at somewhat higher mass flows than that of the unpinched case. The efficiency of the pinched designs is higher over a wide operating range. Only near the stall and choke limits, the efficiencies are almost the same.

The efficiencies at the lower rotational speed $\left(N_{\text {ref }} / N_{\text {des }}=0.95\right)$ are presented in Fig. 4 . At the lower rotational speed, it is hard to distinguish which is the best of the hub and shroud designs, as they all have higher efficiencies than the unpinched one. Of the shroud pinches, the $b / b_{2}=0.854$ is the best, as was the case with the design rotational speed.

At the lower rotational speed, the peak efficiency of the unpinched case is at a lower mass flow than the design mass flow. For the pinched cases, the peak efficiency is at higher mass flows, very close to the design mass flow. There are no major differences in efficiency near the stall limit. 


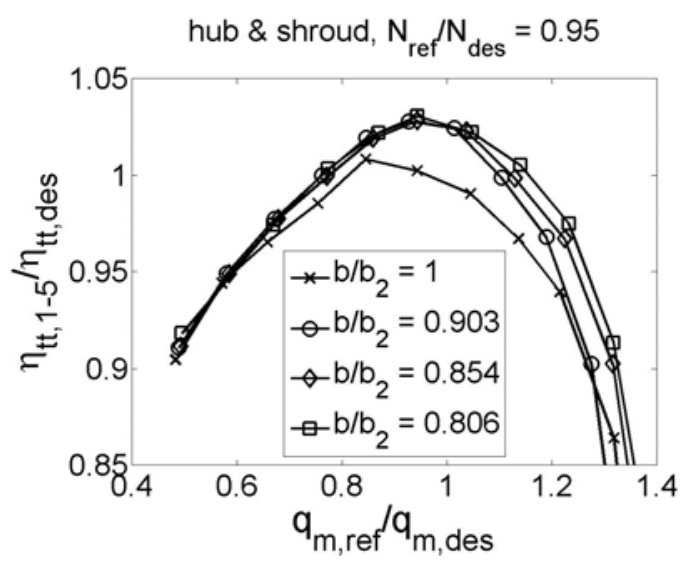

(a)

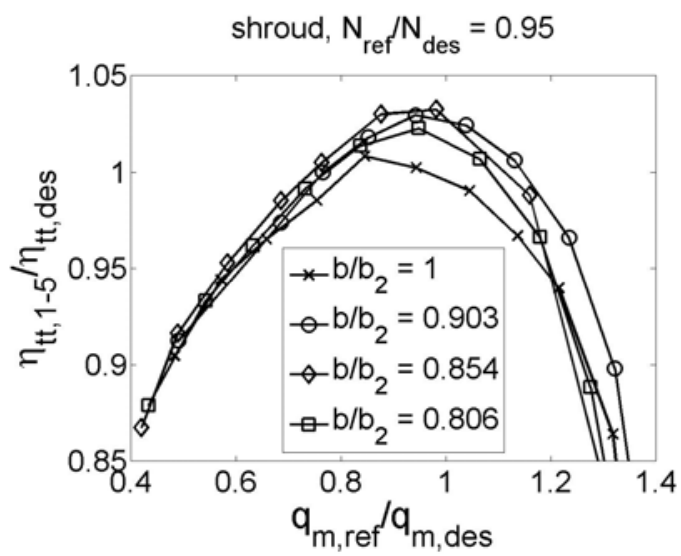

(b)

Figure 5. Total-to-total efficiencies at $N_{\text {ref }} / N_{\text {des }}=0.95$ (a) hub and shroud pinches (b) shroud pinches.

The total-to-total efficiencies at the rotational speed of $N_{\text {ref }} / N_{\text {des }}=1.05$ are presented in Fig. 5 . Also at the higher rotational speed, the pinched designs have better efficiency than the unpinched design. From the hub and shroud pinches, the $b / b_{2}=0.903$ has slightly better efficiency than the other hub and shroud designs. The $b / b_{2}=0.806$ shroud design has lower efficiency than the other two shroud cases. 


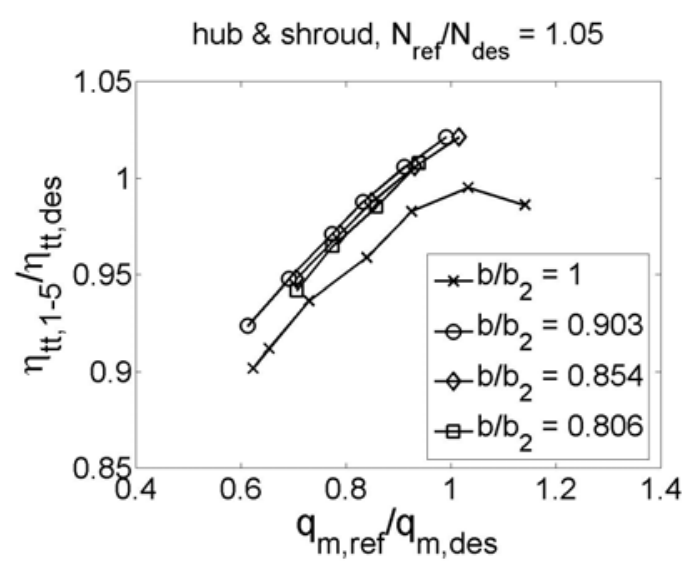

(a)

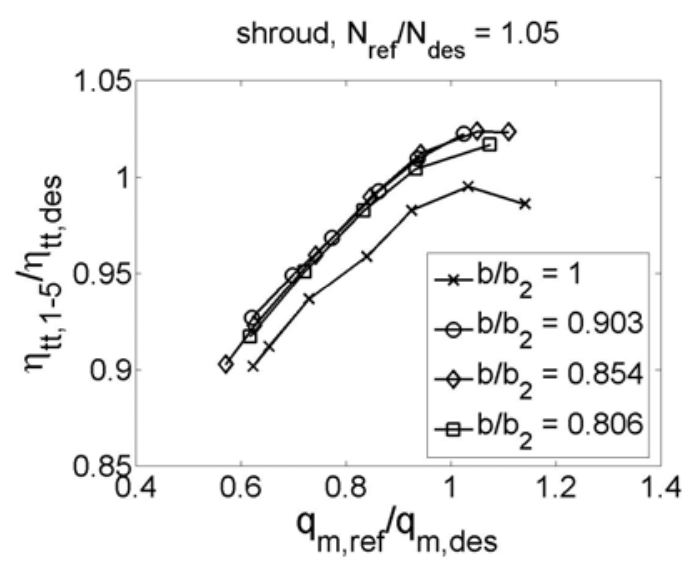

(b)

Figure 6. Total-to-total efficiencies at $N_{\text {ref }} / N_{\text {des }}=1.05$ (a) hub and shroud pinches (b) shroud pinches.

It can be concluded from Figs. 2-5 that the best designs with shroud pinch are the $b / b_{2}=0.903$ and the $b / b_{2}=0.854$, and of the hub and shroud pinches, the $b / b_{2}=0.806$ and the $b / b_{2}=0.854$.

The efficiencies of these designs at the design and lower rotational speeds are presented in Figs. 6 and 7 , respectively.

At both operating speeds, the $b / b_{2}=0.854$ (shroud) is the best of the four, and the $b / b_{2}=0.854$ (hub and shroud) is the worst of the four, so even when the diffuser width is the same, it is more beneficial to implement the pinch to the shroud than to divide it between the hub and the shroud. 
The designs $b / b_{2}=0.806$ hub and shroud, and $b / b_{2}=0.903$ shroud have the same absolute amount of pinch at the shroud. These designs have almost the same efficiencies, so it seems that the pinch at the hub is irrelevant or has only a minor effect on the efficiency.

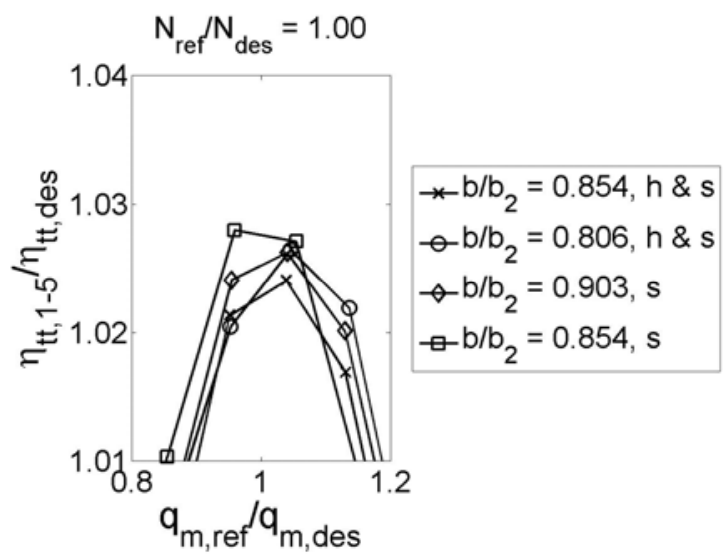

Figure 7. Efficiency of the best designs near the design mass flow at the design rotational speed.

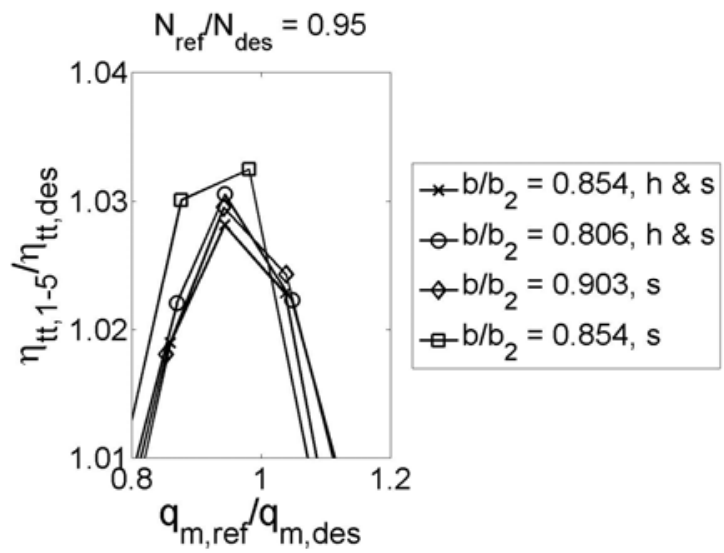

Figure 8. Efficiency of the best designs near the design mass flow at $N_{\text {ref }} / N_{\text {des }}=0.95$.

All in all, this indicates that the shroud pinch is much more beneficial to the efficiency, whereas the hub pinch has only a minor effect or no effect at all. 
The efficiency as a function of the diffuser width at the design rotational speed with two mass flows (the design and 0.8 times the design) is presented in Fig. 8 .

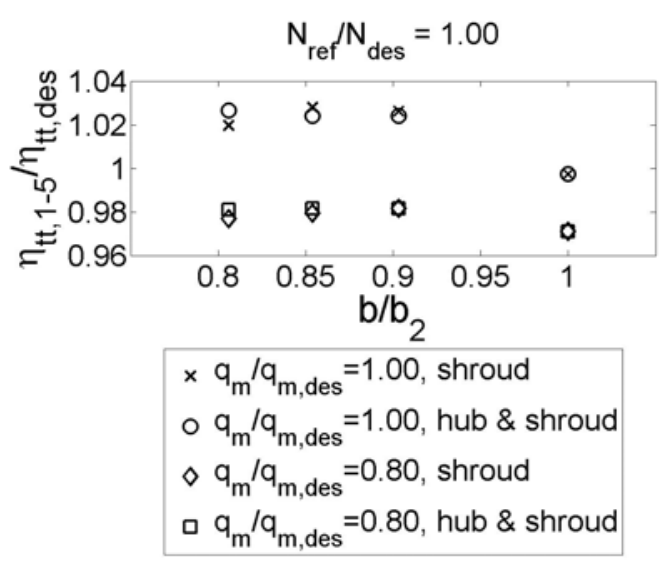

Figure 9. Efficiency as a function of diffuser width at the design rotational speed.

It can be seen that at the design operating point, the shroud pinch increases the efficiency more than the hub and shroud pinch, except for the narrowest diffuser. At the lower mass flow, the hub and shroud pinch is more beneficial.

It can be estimated from Fig. 8 that here the optimum diffuser width ratio is around 0.85 times the impeller exit width, when the reduction is implemented at the shroud. At the lower mass flow, the optimum width ratio is around 0.9 times the impeller exit width.

\subsection{Pressure ratio}

The total-to-total pressure ratios over the whole compressor for the different designs at the design rotational speed are presented in Figs. 9 and 10.

All the pinched cases have a better pressure ratio than the unpinched design over a wide operating range. Only very close to the choke limit, the unpinched design has approximately the 
same pressure ratio. This may indicate that the pinched designs choke at somewhat lower mass flows than the unpinched design. Of the shroud pinched cases, the one with the diffuser width ratio of $b / b_{2}=0.903$ is the best, and of the hub and shroud pinched cases, the $b / b_{2}=0.854$ and $b / b_{2}=$ 0.806 are the best ones, and there is not much difference between the two. The shapes of the pressure ratio curves are similar to all the designs.

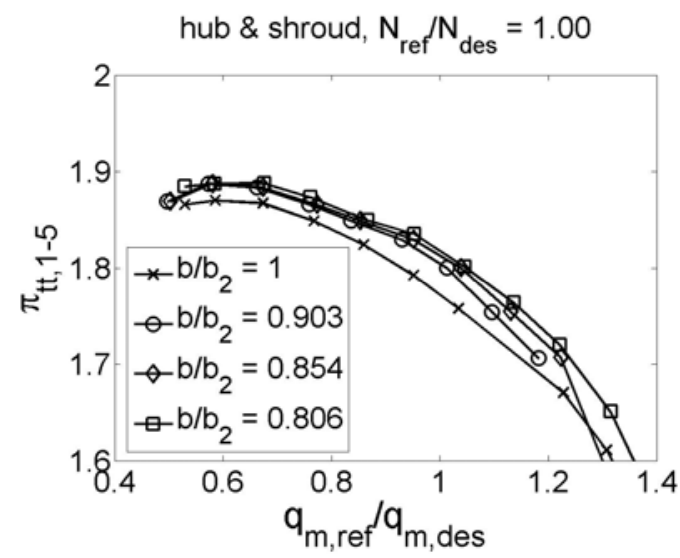

Figure 10. Total-to-total stage pressure ratio at the design rotational speed (hub and shroud pinches).

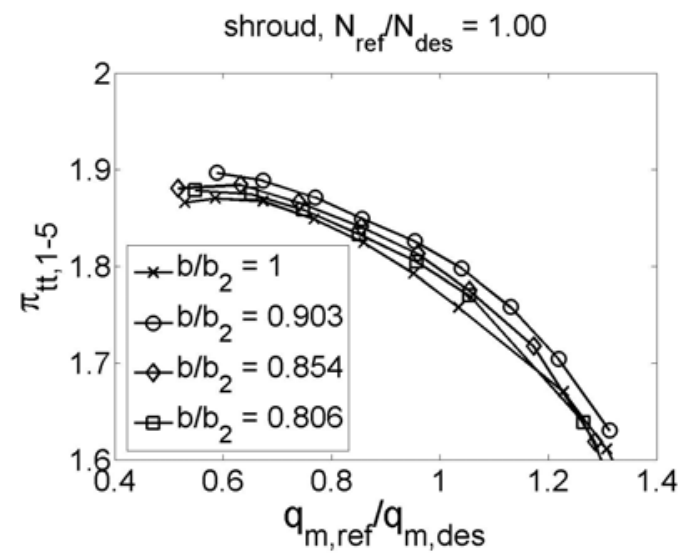

Figure 11. Total-to-total stage pressure ratio at the design rotational speed (shroud pinches). 
The pressure ratios at the lower rotational speed are presented in Fig. 11. At the lower rotational speed, the pressure ratios behave the same way as at the design speed. The best designs are the $b / b_{2}$ $=0.903$ of the shroud pinched design, and $b / b_{2}=0.854$ and $b / b_{2}=0.806$ of the hub and shroud pinched one.

The pressure ratios at the higher rotational speed are presented in Fig. 12. At the higher rotational speed, the results are completely different than at the design and the lower rotational speeds.

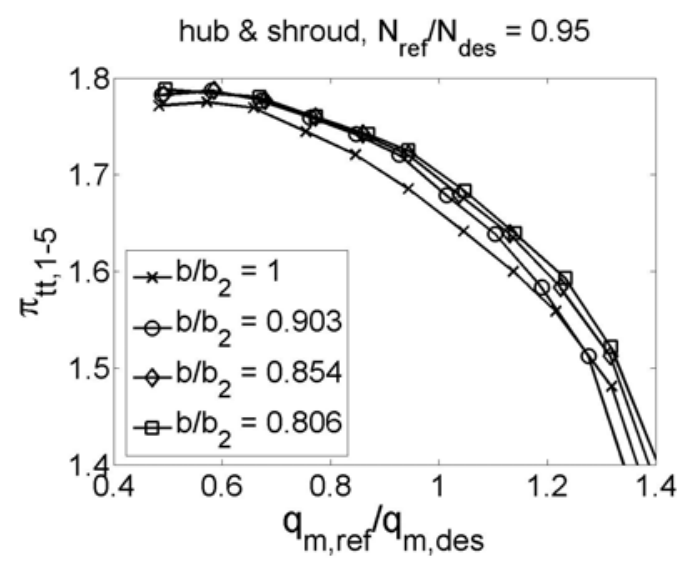

(a)

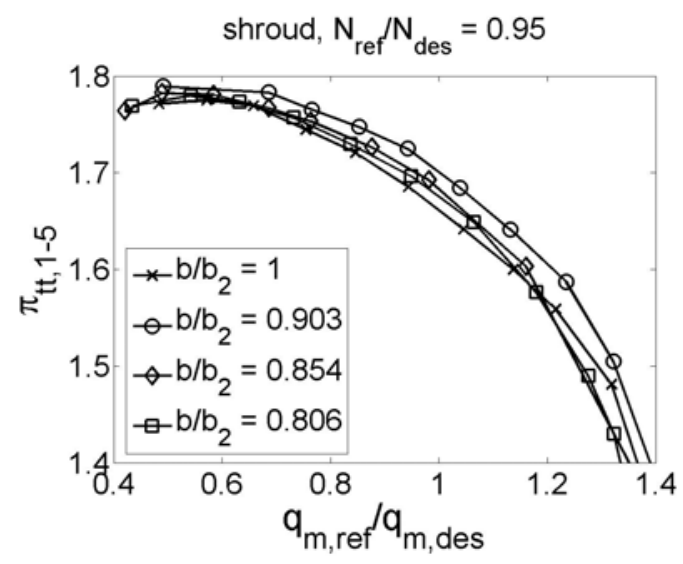

(b)

Figure 12. Stage pressure ratio at $N_{\text {ref }} / N_{\text {des }}=0.95$ (a) hub and shroud pinches (b) shroud pinches. 
The unpinched design has the highest pressure ratio. Of the shroud pinched designs, the one that was the best at the design and lower rotational speeds, has now the worst pressure ratio. The two other designs have almost the same pressure ratios. For the hub and shroud pinched designs, the one that was the best at the design and lower rotational speeds is still the best of the hub and shroud pinched designs, but it has a clearly lower pressure ratio than the unpinched one.

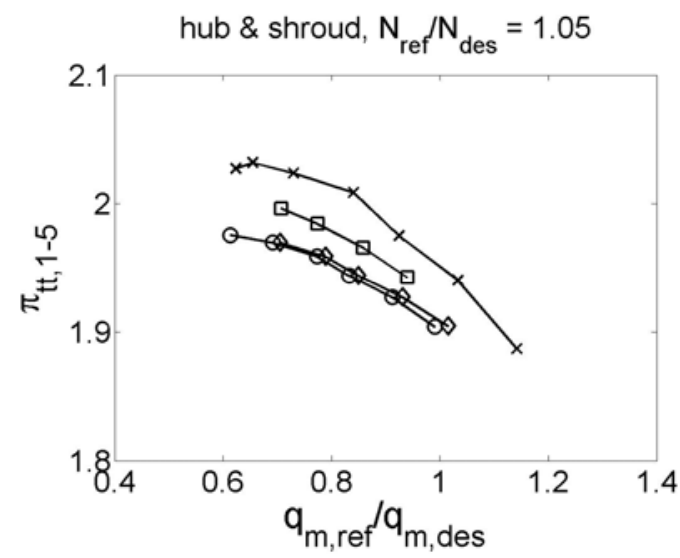

(a)

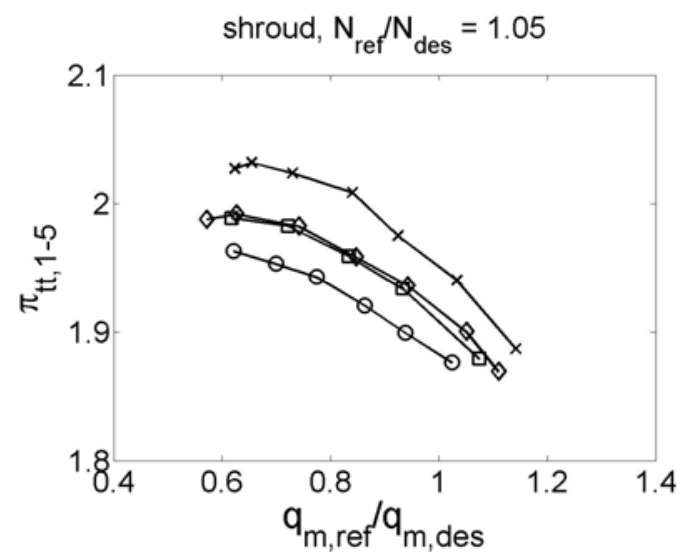

(b)

Figure 13. Stage pressure ratio at $N_{\text {ref }} / N_{\text {des }}=1.05$ (a) hub and shroud pinches (b) shroud pinches. 
It can be concluded from Figs. 9-12 that the best designs at the design and lower rotational speeds with shroud pinch are the $b / b_{2}=0.903$ and the $b / b_{2}=0.854$, and of the hub and shroud pinches the $b / b_{2}=0.806$ and the $b / b_{2}=0.854$, as was the case with efficiency.

The stage pressure ratio of the best designs at the design and the lower rotational speed are presented in Figs. 13 and 14, respectively.

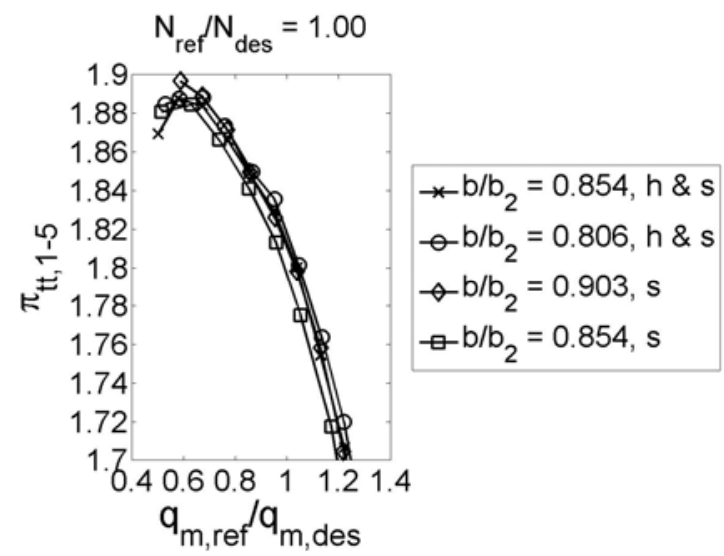

Figure 14. Pressure ratio of the best designs at the design rotational speed.

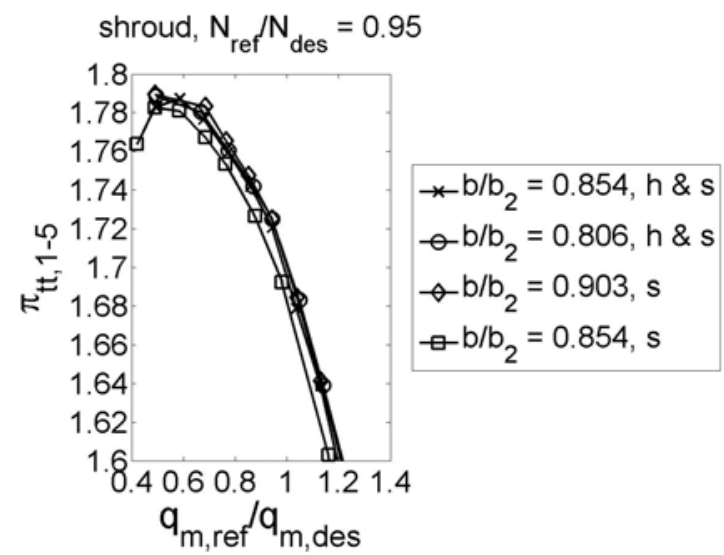

Figure 15. Pressure ratio of the best designs at $N_{\text {ref }} / N_{\text {des }}=0.95$.

At the design and the lower rotational speed, the $b / b_{2}=0.903$ (shroud) and $b / b_{2}=0.806$ (hub and shroud) have the highest pressure ratio, along with the $b / b_{2}=0.854$ (hub and shroud), which 
has marginally lower pressure ratios than the other two. This seems to strengthen the idea that hub pinch has only a minor or negligible effect on the overall performance of the compressor.

Of the four designs, the $b / b_{2}=0.854$ (shroud), which has the highest efficiency, has the lowest pressure ratio.

Better efficiency is possible with constant mass flow only if the pressure ratio is higher, if less work is done to the fluid, or both. Figs. 5 and 12 show that the pinched geometries have better efficiencies, but lower pressure ratios at the higher rotational speed, and hence the work done to the fluid must be less.

We also know that all the work done to the fluid is done in the impeller, so reducing the diffuser width leads to lower work input in the impeller. At the design and lower rotational speeds, both the efficiency and pressure ratios are higher with pinched geometries. However, we can not directly conclude that it is the impeller which performs better also at these rotational speeds, but it seems very plausible.

\section{CONCLUSIONS AND DISCUSSION}

Seven different vaneless diffuser designs were measured, and their overall performance was studied. One of the designs was a basic design with the diffuser width ratio $b / b_{2}$ of 1.0 . The other diffusers had width ratios of $0.903,0.854$ and 0.806 . Three had pinch at the shroud, and three at the hub and at the shroud, divided evenly.

According to the results, it is possible to improve the efficiency and pressure ratios by reducing the diffuser width. The efficiency increased over a wide operating range at all the rotational speeds with all the different pinched diffusers.

The pressure ratio increased over a wide operating range at the design and low rotational speeds, but decreased at the higher rotational speed. Higher efficiency and a lower pressure ratio, observed at the higher rotational speed, are possible only if the impeller work input is smaller. It is plausible that this occurs also at the design and at lower rotational speeds. 
One distinct possibility is that pinch reduces the secondary losses caused by the tip clearance flow. Shroud pinch seems to be more beneficial to the efficiency, which strengthens this point. Also, the worst of the pinched designs was the one with $b / b_{2}=0.903$ (hub and shroud), which had approximately the same amount of pinch at the shroud than what was the compressor tip clearance. It seems that in order to have the possible benefices of the narrower diffuser, the shroud pinch should be greater than the tip clearance, and it is the impeller which performs better.

The increase in the pressure ratio might be caused by smaller boundary layers in the diffuser. The pinch pushes the flow towards the centre of the diffuser, accelerating the flow and decreasing the size of the boundary layer, leading to lower losses in the diffuser.

In most cases, when the shroud pinch was equal, it had no effect if there was also a hub pinch present. This indicates that pinch has indeed an effect on the slow flow region which is present in the centrifugal compressor diffuser near the shroud.

When the diffuser width is reduced, the flow is more radial at the impeller outlet, so the margin to the critical flow angle (stall) is greater. This should make it possible to design more impeller back sweep to increase the efficiency, or a longer diffuser, which should increase the pressure ratio. However, because the effect of pinch is not greatest at lower mass flows, it seems that the radial

velocity is not the defining parameter. In our measurements, the optimum design was the one with the diffuser width ratio $b / b_{2}=0.854$ and reduction implemented only at the shroud. According to the literature, large pinches deteriorate the performance.

\section{ACKNOWLEDGEMENS}

The authors gratefully acknowledge the financial contribution of the Academy of Finland, the Finnish Funding Agency for Technology and Innovation - TEKES, and High Speed Tech Oy Ltd., for their part in financing the research. 


\section{REFERENCES}

[1] Senoo Y., Kinoshita, Y. and Ishida, M. Asymmetric flow in vaneless diffusers of centrifugal blowers. Journal of Fluids Engineering, 1977, 104-114.

[2] Van den Braembuscsche, R. A., Frigne, P., and Roustan, M. Rotating Non-Uniform Flow in Radial Compressors. In Proceedings No 282 AGARD Conference, 1980.

[3] Abidogun, K. B. Effects of Vaneless Diffuser Geometries on Rotating Stall. In IPC2002-27203, $4^{\text {th }}$ International Pipeline Conference, ASME, 2002.

[4] Ludtke, K. Aerodynamic tests on centrifugal process compressors - the influence of vaneless diffuser shape. Journal of Engineering for Power, 1983, 105, 902-909.

[5] Ferrara, G., Ferrari, L., Mengoni, C. P., Lucia, M. D. and Baldassarre, L. Experimental investigation and characterization of rotating stall in a high pressure centrifugal compressor: Part I: Influence of diffuser geometry on stall inception. In proceedings of ASME Turbo Expo, Amsterdam, the Netherlands, 2002, paper GT2002-30389.

[6] Ferrara, G., Ferrari, L., Mengoni, C. P., Lucia, M. D. and Baldassarre, L. Experimental investigation and characterization of rotating stall in a high pressure centrifugal compressor: Part II: Influence of diffuser geometry on stage performance. In proceedings of ASME Turbo Expo, Amsterdam, the Netherlands, 2002, paper GT2002-30390

[7] Cellai, A., Ferrara, G., Ferrari, L., Mengoni, C. P. and Baldassarre, L. Experimental investigation and characterization of rotating stall in a high pressure centrifugal compressor: Part III: Influence of diffuser geometry on stall inception and performance ( $2^{\text {nd }}$ impeller tested). In proceedings of ASME Turbo Expo, 2003, paper GT2003-38390

[8] Di Liberti, J.-L., Wilmsen, B. and Engeda, A. The effect of vaneless diffuser width on the performance of centrifugal compressor. In FED vol. 237, pp 797-803. Fluids Engineering Division Conference, ASME, 1996.

[9] Engeda, A. The influence of diffuser width on the unsteady performance of centrifugal compressor stage. In FEDSM200231250, Fluids Engineering Division Summer Meeting, ASME, 2002.

[10] Turunen-Saaresti, T., Reunanen A. and Larjola J. Computational and experimental study of pinch on the performance of a vaneless diffuser of a centrifugal compressor. Journal of Thermal Science, 2006, 15, 306-313. 
[11] DIN 1952, Durchflußmessung mit genormten Düsen, Blenden un Venturdüsen, Deutsches Institut für Normung e.V. 1971.

[12] ASME PTC 10, ASME Power Test Codes, compressors and exhausters. The American Society of Mechanical Engineering, 1965.

[13] ISO 5389, Turbocompressors - Performance test code. International Standardization Organization, 1992.

[14] VDI 2045 Part 1, Abhname- und Leistungsversuche an Versuchsdurchführung und Garatievergleich (Acceptance and Performance Test on Turbo Compressors and Displacement Compressors, Test Procedure and Comparison with Guaranteed Values). Verein Deutscher Ingenieure, 1993.

[15] VDI 2045 Part 2, Abhname- und Leistungversuche and Verdichetrn, Grundlagen und Beispiele (Acceptance and Performance Test on Turbo Compressors and Displacement Compressors, Theroy and examples). Verein Deutscher Ingenieure, 1993.

\section{APPENDIX 1}

\section{Notation}

diffuser width (m)

$c_{\mathrm{p}} \quad$ isobaric specific heat capacity $(\mathrm{J} / \mathrm{kgK})$

E relative error (\%)

$h \quad$ specific enthalpy $(\mathrm{J} / \mathrm{kg})$

$N \quad$ rotational speed $(1 / \mathrm{s}, \mathrm{rpm})$

$N_{\text {s }} \quad$ specific rotational speed

$p \quad$ pressure $(\mathrm{Pa})$

$q_{\mathrm{m}} \quad$ mass flow $(\mathrm{kg} / \mathrm{s})$

$q_{\mathrm{v}} \quad$ volume flow $\left(\mathrm{m}^{3} / \mathrm{s}\right)$

$R \quad$ specific gas constant $(\mathrm{J} / \mathrm{kgK})$ 


$\begin{array}{ll}R_{\mathrm{H}} & \text { relative humidity }(\%) \\ r & \text { radius }(\mathrm{m}) \\ T & \text { temperature }(\mathrm{K})\end{array}$

Greek alphabet

$\begin{array}{ll}\eta & \text { efficiency } \\ \Omega & \text { angular velocity }(\mathrm{rad} / \mathrm{s}) \\ \pi & \text { pressure ratio }\end{array}$

Subscripts

1 compressor inlet

2 impeller outlet

2' beginning of pinch

3 diffuser outlet

5 compressor outlet

ref reference value

S isentropic

t total

tt total-to-total 